\title{
Perfil da rede de saúde no extremo sul de Santa Catarina: potencialidades e desafios
}

\section{na implantação de um curso de Medicina}

\author{
Profile of the health network in the extreme south of Santa Catarina: potential and challenges in \\ the implementation of a medical course \\ Perfil de la red de salud em el extremo sur de Santa Catarina: potencialidades y desafios em la \\ implementación de um curso médico
}

Recebido: 27/07/2021 | Revisado: 31/07/2021 | Aceito: 02/08/2021 | Publicado: 07/08/2021

Iane Franceschet de Sousa ORCID: https://orcid.org/0000-0002-2061-2851 Universidade Federal de Santa Catarina, Brasil E-mail: iane.franceschet@ufsc.br

Flávia Corrêa Guerra

ORCID: https://orcid.org/0000-0002-3666-0014 Universidade Federal de Santa Catarina, Brasil E-mail: flavia.guerra@ufsc.br

Fábio de Almeida Morais ORCID: https://orcid.org/0000-0001-5039-4034 Universidade Federal de Santa Catarina, Brasil E-mail: fabio.am@ufsc.br

Vanessa Darós De Luca Tomazi ORCID: https://orcid.org/0000-0003-0606-3646 Universidade Federal de Santa Catarina, Brasil E-mail: vanessa.darosdeluca@gmail.com

Mariana Zanette Fernandes ORCID: https://orcid.org/0000-0001-8534-7975 Universidade Federal de Santa Catarina, Brasil E-mail: marizanettef@gmail.com

\begin{abstract}
Resumo
O fortalecimento das parcerias entre os serviços de saúde e as universidades são fundamentais para a consolidação de um sistema de saúde mais resolutivo para a população. Neste sentido, a presente pesquisa objetiva conhecer a estrutura da rede de saúde existente na região do extremo sul de Santa Catarina, relacionando com o nível de complexidade dos serviços oferecidos. Tais serviços fazem parte dos cenários de práticas de ensino dos estudantes do curso de Medicina da UFSC campus de Araranguá, que foi implantado em 2018 e está em processo de consolidação. Foi realizado estudo transversal, nos meses de junho e julho de 2020, através de coleta de dados eletrônicos do DATASUS. Os resultados encontrados demonstraram que, tanto na Atenção Básica quanto na alta e média complexidade, são poucos os serviços oferecidos e/ou baixo número de profissionais qualificados para atender a demanda da região da Amesc. Conclui-se que a entrada de estudantes de Medicina e, posteriormente, dos egressos, a médio e longo prazo, resultará no fortalecimento do sistema de saúde local.
\end{abstract}

Palavras-chave: Acesso universal aos serviços de saúde; Sistema Único de Saúde; Educação médica.

\begin{abstract}
Strengthening partnerships between health services and universities are fundamental for the consolidation of a health system that is more effective for the population. In this sense, this research aims to understand the structure of the existing health network in the extreme south region of Santa Catarina, relating to the level of complexity of the services offered. Such services are part of the teaching practice scenarios for students of the UFSC Medicine course at the Araranguá campus, which was implemented in 2018 and is in the process of consolidation. A cross-sectional study was carried out in the months of June and July 2020, through the collection of electronic data from DATASUS. The results found showed that, both in Primary Care and in the high and medium complexity, there are few services offered and / or low number of qualified professionals to meet the demand in the Amesc region. It is concluded that the entrance of medical students and, later, of the graduates, in the medium and long term, will result in the strengthening of the local health system.
\end{abstract}

Keywords: Universal access to health care services; Unified Health System; Education, medical. 


\begin{abstract}
Resumen
El fortalecimiento de las alianzas entre los servicios de salud y las universidades es fundamental para la consolidación de un sistema de salud más efectivo para la población. En este sentido, esta investigación tiene como objetivo comprender la estructura de la red de salud existente en el extremo sur de Santa Catarina, relacionándola con el nivel de complejidad de los servicios ofrecidos. Dichos servicios forman parte de los escenarios de práctica docente para los estudiantes de la carrera de Medicina de la UFSC en el campus de Araranguá, que se implementó en 2018 y se encuentra en proceso de consolidación. Se realizó un estudio transversal en los meses de junio y julio de 2020 , mediante la recolección de datos electrónicos de DATASUS. Los resultados encontrados mostraron que, tanto en Atención Primaria como en la complejidad alta y media, hay pocos servicios ofertados y / o bajo número de profesionales calificados para atender la demanda en la región Amesc. Se concluye que el ingreso de los estudiantes de medicina y, posteriormente, de los egresados, en el mediano y largo plazo, redundará en el fortalecimiento del sistema local de salud.
\end{abstract}

Palabras clave: Acceso universal a los servicios de salud; Sistema Único de Salud; Educación médica.

\title{
1. Introdução
}

Diante da insuficiência de recursos humanos capacitados para atender às necessidades de saúde da população brasileira, a formação acadêmica dos profissionais de saúde tornou-se objeto de frequentes reflexões (Mendes et al., 2020). Por isso, nas últimas décadas, vem se construindo, dentro da formação médica, uma relação de aproximação entre ensino e serviço, como reflexo de uma série de movimentos na direção de transformações dos modelos tradicionais de ensino para formação na saúde, os quais tem se mostrado incapazes de responder adequadamente às necessidades apresentadas pela população.

Esses movimentos vêm na tentativa contrária ao modelo hegemônico flexneriano, centrado em conteúdos compartimentados e isolados, que fragmentam o ser humano em especialidades, dissociando os conhecimentos das áreas básicas e clínicas, foca o processo de ensino-aprendizagem no espaço da prática hospitalar, medicaliza o social, orienta para o mercado, incentiva a especialização precoce e a incorporação tecnológica, a partir de bases pedagógicas tradicionais, perpetuando, assim, o modelo vigente e ineficiente de práticas em saúde (Ceccim \& Feuerwerker, 2004; Mendes et al., 2020).

Apesar da importância das reformas de Flexner para a educação médica, a ênfase no modelo biomédico, centrado na doença e no hospital, conduziu os programas educacionais médicos a uma visão reducionista. Ao adotar o modelo de saúde unicausal, biologicista, a proposta de Flexner reserva pequeno espaço (se algum), para as dimensões psicológica, social e econômica.

Buscando mudar essa perspectiva, os cursos de Medicina, respaldados nas novas Diretrizes Curriculares Nacionais (DCN) de 2014, devem vincular a formação médico-acadêmica às necessidades sociais de saúde, com ênfase no SUS, por meio da integração ensino-serviço (Resolução no 3, 2014). Porém, Francischetti, Holzhausen e Peters (2020) enfatizam que um ponto frágil em alguns currículos de cursos de Medicina é a falta de interação precoce do estudante no cenário de prática, pois é justamente esta aproximação com a prática que vai gerar o "aprender contextualizado".

A articulação entre ensino e serviço vem sendo enfatizada pelo Ministério da Saúde e Ministério da Educação, como uma estratégia importante para a formação de profissionais que atendam aos princípios e diretrizes do SUS. Mais recentemente, uma nova demanda foi lançada aos municípios através dos Contratos Organizativos de Ação Pública EnsinoSaúde (COAPES), visando o fortalecimento da integração entre ensino, serviços e comunidade no âmbito do SUS (Portaria Interministerial $\left.\mathrm{n}^{\mathrm{o}} 1.127,2015\right)$.

Desta forma, faz-se necessário pensar a mudança na formação em saúde a partir da discussão sobre a articulação entre as universidades e os serviços de saúde. Esta articulação permite a compreensão das necessidades de saúde da população, além de direcionar a formação para o Sistema Único de Saúde (SUS), que será novamente beneficiado com a inserção desses futuros profissionais na rede de serviços (Mendes et al., 2020).

A preceptoria na atenção primária à saúde envolve aspectos peculiares, pois ao adentrar no serviço de saúde o aprendiz se depara com profissionais muitas vezes desmotivados, desinformados, organização do processo de trabalho 
comprometida, dentre outros. Nesse sentido, a aproximação do cenário formativo ao cenário de práticas de trabalho pode ser propulsora da oxigenação do serviço favorecendo as trocas, teórica e prática, para melhorias da atenção ao usuário (Barreto, Monteiro, Magalhães, Almeida \& Souza, 2011).

Considerando as mudanças globais do ensino médico, o Brasil tem demonstrado esforços para alcançar os níveis de qualidade destacados nos principais documentos internacionais, os quais estabelecem diretrizes e critérios para nortear a formação médica, como o Global Standards in Medical Education, o Canadian Medical Education Directions for Specialists (CanMEDS), a Association for Medical Education in Europe (AMEE), entre outros (Frank, Snell \& Sherbino, 2015; Word Federation for Medical Education [WFME], 2003; Francischetti et al, 2020). Entretanto, devido às diferenças regionais no Brasil, alguns locais apresentam maior fragilidade em relação ao sistema de saúde, em detrimento a outros mais desenvolvidos. O fato é que as regiões menos favorecidas sofrem com a precariedade do sistema, dificultando avanços inclusive na formação, impondo desafios às universidades que necessitam da rede para a formação dos egressos de Medicina.

\subsection{Contextualização da região do extremo sul catarinense: características socioeconômicas e de saúde}

O município de Araranguá está localizado na região do Extremo Sul Catarinense, fazendo parte desta região, outros 14 municípios: Balneário Arroio do Silva, Balneário Gaivota, Ermo, Jacinto Machado, Maracajá, Meleiro, Morro Grande, Passo de Torres, Praia Grande, Santa Rosa do Sul, São João do Sul, Sombrio, Timbé do Sul e Turvo. Os 15 municípios constituem a microrregião administrativa da AMESC (Associação dos Municípios do Extremo Sul de Santa Catarina). Segundo dados do IBGE/2019, a população residente estimada na região é de 202.376 habitantes, sendo que o maior município é Araranguá com 68.228 habitantes, e o menor município é Ermo, com 2.063 habitantes (Instituto Brasileiro de Geografia e Estatística [IBGE], 2019).

A região caracteriza-se como uma das mais pobres de Santa Catarina, com índice de desenvolvimento humano e índice per capita anual inferiores aos do estado e do país. Segundo dados do IBGE relacionados ao Mapa de Pobreza e Desigualdade dos Municípios Brasileiros - 2003, a incidência de pobreza em Araranguá atingia naquele ano 27,9\% da população do município. Adicionalmente, o Índice de Desenvolvimento Humano de Araranguá, que se trata de uma medida resumida do progresso em longo prazo, em três dimensões básicas do desenvolvimento humano (renda, educação e saúde) alcançou em 2010 o valor de 0,760, colocando o município na $38^{\text {a }}$ posição estadual. É importante destacar que no que diz respeito à dimensão educação, Araranguá ocupava a 63ª posição nesse mesmo ano. Araranguá possuía em 2017 um PIB per capita da ordem de $\mathrm{R} \$ 25.697,19$, colocando o município na $183^{\text {a }}$ posição do ranking estadual (IBGE, 2019).

A taxa bruta de natalidade em 2010 no município de Araranguá era de 14,5 nascidos por mil habitantes, sendo maior que a taxa média de Santa Catarina e menor que a do Brasil. A taxa de mortalidade infantil em 2015 foi de 12,92 óbitos/1.000 nascidos vivos contra 13,82 óbitos/1.000 nascidos vivos no país (Sistema de Informação sobre Mortalidade [SIM], 2012).

A esperança de vida ao nascer em Araranguá, que consiste no número médio de anos que um grupo de indivíduos nascido no mesmo ano pode esperar viver, se mantidas desde o seu nascimento as taxas de mortalidade observadas naquele ano, foi de 77,7 anos. Esse número foi menor que aquele observado no estado (78,7 anos) e maior que o observado no Brasil (75,5 anos) (IBGE, 2019).

\subsection{O contexto do nascimento do curso de Medicina na UFSC campus de Araranguá}

Considerando a Universidade como lócus privilegiado para reflexão e construção de conhecimento, além de importante propulsora do desenvolvimento regional, em 2018 foi implantado o curso de Medicina na UFSC, campus de Araranguá. O curso de Medicina nasceu através do Programa Mais Médicos do Brasil (PMMB), instituído pela Lei no 12.871, de 22 de outubro de 2013 (Lei n 12.871, 2013). Essa lei, em seu Art. 2o, explicita que, para a consecução dos seus objetivos, 
serão adotadas, entre outras, a reordenação da oferta de cursos de Medicina e de vagas para residência médica, priorizando regiões de saúde com menor relação de vagas e médicos por habitante; o estabelecimento de novos parâmetros para a formação médica no país; e o aperfeiçoamento de médicos na área de atenção básica em saúde, mediante integração ensino-serviço, inclusive por meio de intercâmbio internacional nessas regiões (Pedrosa, 2019).

Desde então, tem sido um desafio implantar um curso de Medicina em um campi fora da sede universitária. O campus de Araranguá foi criado a partir do Programa de Apoio a Planos de Reestruturação e Expansão das Universidades Federais Brasileiras (REUNI), considerado uma das ações integrantes ao Plano de Desenvolvimento da Educação (PDE). Foi instituído em reconhecimento ao papel estratégico das universidades, em especial do setor público, para o desenvolvimento econômico e social, sobretudo das regiões mais carentes (Decreto $\mathrm{n}^{\circ}$ 6.096, 2007).

Somado-se às dificuldades técnico-administrativa institucionais, a implantação do curso de Medicina em Araranguá envolveu disputas políticas e sociais, inclusive na pactuação da parceria com a rede de saúde local. No entanto, enfatiza-se que o curso de Medicina da UFSC Araranguá veio na perspectiva da descentralização da formação e da fixação de profissionais na região com a intencionalidade de melhorar o acesso aos serviços de saúde. Conforme aponta Pedrosa (2019), esta é considerada uma intervenção com características inovadoras.

Como ocorre em outros cursos de Medicina que nasceram com o PMMB, a inovação coloca-se como desafio em diversas instâncias, como a utilização de currículos integrados, a incorporação de métodos de ensino-aprendizagem centrados nos estudantes e a busca por uma maior integração com equipamentos sociais, serviços de saúde e comunidade em geral (Oliveira, Melo, Pinto, Azevedo \& Santos, 2017; Pedrosa, 2019).

Em relação à interação universidade - rede de saúde, o curso de Medicina apresenta um Projeto Pedagógico estruturado com um eixo de interação comunitária, que está organizado de maneira vertical, do 1 o ao 40 ano do curso. Isso se traduz na inserção precoce do estudante na rede de serviços, conforme preconizam as DCN (Resolução no 3, 2014). Este fato vem ao encontro das demandas nacionais no que tange à mudança do paradigma hospitalocêntrico para a compreensão dos modelos assistenciais como um todo, utilizando para o alcance desses objetivos, as metodologias ativas de ensinoaprendizagem como estratégia.

Ainda, de acordo com as DCN, 35\% da carga-horária do curso de Medicina deve ser alocada para o internato médico que, conforme o Projeto Pedagógico do Curso de Medicina ocorre no 5o e 60 ano. O Estágio Curricular Obrigatório de formação em serviço, em regime de internato, deve ocorrer sob supervisão, em serviços próprios, conveniados ou em regime de parcerias estabelecidas por meio de Contrato Organizativo da Ação Pública Ensino-Saúde (COAPES) com as Secretarias Municipais e Estaduais de Saúde (Resolução nº 3, 2014).

A preceptoria exercida por profissionais do serviço de saúde tem supervisão de docentes da universidade, aproximando os laços entre o ensino e a rede de saúde. O mínimo de 30\% da carga horária prevista para o internato médico da Graduação em Medicina é desenvolvido na Atenção Básica e em Serviço de Urgência e Emergência do SUS, privilegiando a área da Medicina Geral de Família e Comunidade. Os 70\% da carga horária restante do internato incluirão, necessariamente, aspectos essenciais das áreas de Clínica Médica, Cirurgia, Ginecologia-Obstetrícia, Pediatria, Saúde Coletiva e Saúde Mental, em atividades eminentemente práticas (Resolução nº 3, 2014).

Portanto, conforme preconizado pelas DCN, tanto a Atenção Primária quanto a média e alta complexidade contemplam os cenários de prática para os estudantes de Medicina. A presença da universidade incentiva, além do ensino, a pesquisa e extensão na região, como forma de estímulo à melhoria dos serviços ofertados à população. Ainda, a presença da universidade vincula os profissionais do serviço na formação dos egressos, com as atividades de preceptoria (Resolução $n^{\circ} 3$, 2014).

Pesquisa realizada com os profissionais de saúde atuantes no município de Araranguá revelaram que há interesse em 
cursos na área de saúde pública e outros assuntos voltados para o serviço. Isto reforça a necessidade de fortalecimento do programa de educação permanente no município, voltado à qualificação para o serviço e para a necessidade de saúde das pessoas. Também reforça a importância da integração ensino-serviço-comunidade, por meio da inserção de docentes e estudantes da graduação na rede pública de saúde, visando à formação profissional com uma abordagem integral do processo saúde-doença com ênfase na atenção básica (Martins et al., 2020; Schipley \& Cunningham, 2001).

Martins et al. (2020) relatam, ainda, que em relação a políticas de educação permanente, o município de Araranguá executa um Programa de Educação Permanente na Atenção Básica, organizado pela própria secretaria de saúde do município, cujo cronograma prevê reuniões mensais com os profissionais de saúde. Os temas discutidos são demandas levantadas pelos próprios profissionais, incluindo assuntos nas áreas de Saúde da Mulher, Doenças Infectocontagiosas, Tabagismo, álcool e outras drogas, saúde mental, curativos, vacinação, regulação das filas, entre outros.

Neste sentido, pelo fato do curso de Medicina da UFSC Araranguá encontrar-se em processo de implantação e por necessitar da parceira da rede de saúde loco regional para se consolidar, é essencial conhecer a estrutura da rede de saúde existente na região da AMESC, relacionando com o nível de complexidade dos serviços oferecidos, os quais fazem parte dos cenários de prática de ensino por meio da integração ensino-serviço-comunidade.

\section{Metodologia}

Estudo observacional, descritivo, transversal, com abordagem quantitativa. De acordo com Rouquayrol e Gurgel (2013), nos estudos transversais as mensurações são feitas em um único momento no tempo e objetivam descrever a prevalência do objetivo de pesquisa.

A coleta de dados foi realizada no mês de junho de 2020, por meio do acesso à base de dados do DATASUS, no Cadastro Nacional dos Estabelecimentos de Saúde (CNES). A pesquisa na base cadastral do CNES foi realizada por município, através da identificação dos estabelecimentos de saúde existentes em cada nível de gestão: municipal, estadual e federal. Posteriormente, foram categorizados conforme nível de complexidade de atendimento: Atenção Primária, média complexidade e alta complexidade.

A coleta de dados foi realizada por acadêmicas do curso de Medicina, sendo que os dados foram armazenados e analisados através do programa Microsoft Excel e expostos no formato de planilhas. Como os dados foram compilados apenas a partir de informações de domínio público, sem identificação de participantes da pesquisa e sem envolvimento de seres humanos, não houve a necessidade de aprovação pelo Comitê de Ética em Pesquisa com Seres Humanos (Resolução no 466, 2012).

\section{Resultados e Discussão}

As informações referentes à rede de saúde dos 15 municípios pertencentes à região da AMESC, segundo dados cadastrados no CNES, no mês de junho de 2020, foram organizados em quadros e categorizados conforme o nível de complexidade. Os resultados estão estruturados de acordo com a os níveis de complexidade: Estrutura da rede de Atenção Básica e Estrutura da rede de média e alta complexidade.

\subsection{Estrutura da rede de Atenção Básica de Saúde}

Os resultados demonstraram que todos os municípios possuem uma estrutura física administrativa para as dependências da secretaria de saúde, tendo em anexo a esta estrutura a central de regulação e a farmácia básica. Além disso, alguns municípios possuem estrutura física da clínica de fisioterapia e academias da saúde. Todos os municípios possuem estruturas físicas de Unidades Básicas de Saúde, variando de 1 UBS em município de menor porte, até 16 UBS em Araranguá, 
que é o município de maior porte da região. O Quadro 1 demonstra a organização da rede de serviços de saúde na Atenção Primária.

Quadro 1 - Descrição da rede de serviços de Atenção Primária à Saúde dos municípios da região da AMESC.

\begin{tabular}{|c|c|c|c|}
\hline Município & UBS & Academia da saúde & Clínica de fisioterapia \\
\hline Araranguá & 16 & 2 & 1 \\
\hline Bal. Arroio do Silva & 3 & - & 1 \\
\hline Bal. Gaivota & 4 & - & - \\
\hline Ermo & 1 & 1 & 1 \\
\hline Jacinto Machado & 8 & 1 & - \\
\hline Maracajá & 1 & - & - \\
\hline Meleiro & 4 & - & - \\
\hline Morro Grande & 2 & - & - \\
\hline Passo de Torres & 2 & - & - \\
\hline Praia Grande & 3 & 1 & - \\
\hline Santa Rosa & 5 & - & - \\
\hline São João do Sul & 4 & 1 & - \\
\hline Sombrio & 8 & - & - \\
\hline Timbé do Sul & 2 & 1 & - \\
\hline Turvo & 6 & 1 & 1 \\
\hline TOTAL & 69 & 8 & 4 \\
\hline
\end{tabular}

Fonte: Autores.

Em relação ao quantitativo de UBS existentes na região, de aproximadamente 70 unidades, estas comportam a maior parcela dos estudantes de Medicina na fase de estágio obrigatório no formato de internato médico. Entretanto, é necessário averiguar quais unidades de saúde comportam, além dos internos, a inserção precoce dos estudantes, a qual também é recomendada pelas DCN (Resolução $\left.n^{\circ} 3,2014\right)$.

Ainda, há necessidade de pactuação com toda a rede de saúde da Amesc a parceria entre os municípios e a universidade, sendo adequado a formalização via Contratos Organizativos de Ação Pública Ensino-Saúde (Coapes) (Portaria Interministerial $\mathrm{n}^{\mathrm{o}}$ 1.127, 2015; Brasil, 2018).

Neste contrato, deve estar relacionado o conjunto de serviços de saúde sob gestão municipal que recebe os estudantes de medicina e/ou residentes, bem como as atribuições de ambas as partes, serviço de saúde e instituição de ensino. Além disso, é importante constar no COAPES a descrição das atividades em relação à assistência aos usuários do SUS, ao ensino dos estudantes e residentes, o papel da gestão local, propostas de contribuições das instituições de ensino para processos de educação permanente dos gestores e trabalhadores dos estabelecimentos de saúde, bem como as diretrizes para pesquisa e atividades de extensão que sejam de interesse das instituições de ensino e de saúde (Ministério da Saúde, 2018). 
Atualmente, milhares de estudantes da área de saúde, inclusive estudantes de medicina e residentes, usam as unidades do SUS como cenários de práticas. Por isso a necessidade de planejar e pactuar de forma clara as responsabilidades bilaterais. O COAPES pretende contribuir para aperfeiçoar a integração ensino e serviço, além de preparar as condições para o novo contexto da graduação de medicina e residência médica (Ministério da Saúde, 2018).

Devido a caracterização da região da Amesc como uma das menos desenvolvidas do estado de Santa Catarina, o curso de Medicina em Araranguá se destaca pela necessidade de fixação de médicos na região. A maioria dos municípios é de pequeno porte e é caracterizado como zona rural, sendo este um ponto norteador das políticas educacionais para enfrentamento das desigualdades no acesso ao cuidado, especialmente na Atenção Básica.

Estudo publicado por Rocha, Boiteux, Azevedo, Siqueira e Andrade (2020) aponta que o vínculo com a região é o critério isolado mais importante, corroborando evidências prévias da literatura sobre o tema. É importante ressaltar que esse vínculo se caracteriza tanto pela naturalidade do estudante quanto pelo tempo de residência e/ou de ter cursado o ensino médio nessas áreas.

Evidências de que a exposição rural durante a graduação médica (ciclo básico e internato) aumentou a probabilidade de trabalho em área rural, com médias acima das nacionais (de 26\% a 67\%). A duração das atividades em áreas rurais foi apontada como importante fator, porém poucos estudos (três) mostraram uma proporção entre o aumento do tempo de experiências rurais e o aumento da fixação (Rocha et al., 2020).

Concomitantemente a consolidação da graduação, as DCN enfatizam a necessidade de implantar programas de residência médica em Medicina de Família e Comunidade (MFC), em consonância com as diretrizes do Programa Mais Médicos do Brasil (Resolução nº 3, 2014; Lei nº 12.871, 2013).

A residência médica é o modelo de especialização médica em serviço, consagrado no Brasil e em todo o mundo, reconhecido por oferecer uma formação que visa garantir os melhores padrões assistenciais à saúde da população dentro das competências e atribuições de cada especialidade médica em um sistema universal de saúde. Os Programas de Residência Médica de Medicina de Família e Comunidade devem integrar a política de provimento do "Médicos pelo Brasil", servindo como meio de garantir formação de qualidade na especialidade e promover a consolidação da Atenção Primária em Saúde (Sociedade Brasileira de Medicina de Família e Comunidade [SBMFC], 2019).

Somado-se a isso, as DCN dos cursos de graduação em Medicina estabelecem que pelo menos $30 \%$ da carga horária prevista para o internato médico deve ser desenvolvido na Atenção Básica e em Serviço de Urgência e Emergência do SUS (Resolução $n^{\circ} 3$, 2014). Isso corrobora para a importância em utilizar a rede de saúde existente na região da Amesc, e possivelmente expandir para municípios de outras regiões mais próximas.

\subsection{Estrutura da rede de média e alta complexidade}

A estrutura hospitalar da região da Amesc contempla 7 hospitais de pequeno e médio porte e 1 policlínica. A relação dos hospitais pode ser visualizada no Quadro 2. 
Quadro 2 - Descrição da estrutura hospitalar existente na região da AMESC em relação ao número de leitos totais e leitos destinados ao SUS.

\begin{tabular}{|c|c|c|c|}
\hline Município & Hospital & Número de leitos totais & Número de leitos SUS \\
\hline Araranguá & Hospital Regional de Araranguá & 137 & 137 \\
\hline Jacinto Machado & Hospital São Roque & 45 & 36 \\
\hline Meleiro & Hospital São Judas Tadeu & 50 & 49 \\
\hline Praia Grande & Hospital Nossa Senhora de Fátima & 50 & 105 \\
\hline Sombrio & Hospital Dom Joaquim & 107 & 21 \\
\hline Timbé do Sul & Hospital Santo Antônio & 28 & 36 \\
\hline Turvo & Hospital São Sebastião & 60 & $\mathbf{4 1 4}$ \\
\hline TOTAL & $\mathbf{7}$ Hospitais & $\mathbf{4 7 7}$ & \\
\hline
\end{tabular}

Fonte: Autores.

Em relação ao perfil dos hospitais, 5 possuem gestão estadual e 2 gestão mista. Os que possuem gestão mista são os hospitais dos municípios de Meleiro e Turvo. Ademais, esses sete hospitais têm a natureza jurídica caracterizada como entidade sem fins lucrativos.

No total, existem 477 leitos hospitalares em atividade na região da Amesc. Destes, um montante de 414 leitos atende exclusivamente pacientes internados pelo SUS.

Dentre os hospitais da região da AMESC, o maior deles é o Hospital Regional de Araranguá (HRA), cujo financiamento é estadual com contrato de gestão terceirizado por uma entidade sem fins lucrativos. É o único hospital que presta a totalidade de atendimentos pelo SUS e serve como referência regional para atendimento, sendo o de maior porte em relação ao número de leitos e de serviços especializados. Juntamente com o HRA funciona, desde 2018, a Policlínica Regional da região da AMESC, ampliando os serviços oferecidos à população.

A Policlínica Regional também recebe financiamento estadual com contrato de gestão terceirizado com a mesma entidade sem fins lucrativos que administra o HRA. O hospital e a policlínica disponibilizam consultas e procedimentos ambulatoriais para usuários egressos do próprio HRA, bem como demais pacientes encaminhados pelas Centrais de Regulação do Estado e do Município de Araranguá, de acordo com prévia pactuação (Secretaria do Estado [SES], 2020).

A disponibilidade de consultas médicas, por parte do hospital e policlínica, são as seguintes: Clínica Cirúrgica (Anestesiologia, Cirurgia Bucomaxilofacial, Cirurgia Geral, Cirurgia Vascular, Oftalmologia, Ortopedia, Otorrinolaringologia, Proctologia e Urologia); Clínica Médica (Cardiologia, Endocrinologia, Gastroenterologia, Nefrologia. Neurologia e Pneumologia); Clínica Gineco-Obstétrica (Obstetrícia, Ginecologia e Mastologia); Clínica Pediátrica (Cirurgia Pediátrica). Nos procedimentos ambulatoriais prestados estão os em Oftalmologia e em outras especialidades (SES, 2020).

Dentre os serviços oferecidos pelos estabelecimentos listados no Quadro 2, enquadram-se como média complexidade ambulatorial. A média complexidade ambulatorial é composta por ações e serviços que visam atender aos principais problemas e agravos de saúde da população, cuja complexidade da assistência na prática clínica demande a disponibilidade de profissionais especializados e a utilização de recursos tecnológicos, para o apoio diagnóstico e tratamento (Brasil, 2004).

Já a atenção especializada é definida como de alta complexidade, cujo conjunto de procedimentos envolve alta tecnologia e alto custo, objetivando propiciar à população acesso a serviços qualificados, integrando-os aos demais níveis de 
atenção à saúde (atenção básica e de média complexidade) (Brasil, 2004).

Neste sentido, a região da Amesc concentra nos estabelecimentos de saúde listados no Quadro 2, enquadrando-se dentro do alcance da média complexidade.

A região da Amesc ainda conta com uma Unidade de Pronto Atendimento (UPA) que presta atendimento para os 15 municípios, além de outros que também existem nas proximidades. A UPA está localizada no município de Araranguá, cuja secretaria de saúde faz a gestão e o financiamento.

Portanto, os estágios na área de urgência e emergência, conforme previstos nas DCN dos cursos de graduação em Medicina, contam com alguns estabelecimentos de saúde da região, como a UPA de Araranguá e os serviços de prontoatendimento hospitalares.

A seguir, o Quadro 3 apresenta o detalhamento dos leitos hospitalares existentes na região, em relação a área de cobertura por especialidade médica. 
Research, Society and Development, v. 10, n. 10, e139101018767, 2021

(CC BY 4.0) | ISSN 2525-3409 | DOI: http://dx.doi.org/10.33448/rsd-v10i10.18767

Quadro 3 - Descrição detalhada do perfil de leitos hospitalares por especialidade médica.

\begin{tabular}{|c|c|c|c|c|c|c|c|c|}
\hline \multirow{3}{*}{$\begin{array}{c}\text { Municípios / } \\
\text { Hospitais }\end{array}$} & \multicolumn{8}{|c|}{ Leitos por especialidade médica } \\
\hline & Araranguá & $\begin{array}{c}\text { Jacinto } \\
\text { Machado }\end{array}$ & Meleiro & Praia Grande & Sombrio & Timbé do Sul & Turvo & TOTAL \\
\hline & $\begin{array}{l}\text { Hospital } \\
\text { Regional } \\
\text { de } \\
\text { Araranguá }\end{array}$ & $\begin{array}{l}\text { Hospital } \\
\text { São } \\
\text { Roque }\end{array}$ & $\begin{array}{l}\text { Hospital } \\
\text { São } \\
\text { Judas } \\
\text { Tadeu }\end{array}$ & $\begin{array}{l}\text { Hospital } \\
\text { Nossa } \\
\text { Senhora de } \\
\text { Fátima }\end{array}$ & $\begin{array}{l}\text { Hospital } \\
\text { Dom } \\
\text { Joaquim }\end{array}$ & $\begin{array}{l}\text { Hospital Santo } \\
\text { Antônio }\end{array}$ & $\begin{array}{l}\text { Hospital } \\
\text { São } \\
\text { Sebastião }\end{array}$ & \\
\hline UTI Adulto & 10 & - & - & - & - & - & - & 10 \\
\hline UTI Covid 19 & 10 & - & - & - & - & - & - & 10 \\
\hline Cirurgia Geral & 15 & 7 & 2 & 7 & 30 & 5 & 5 & 71 \\
\hline $\begin{array}{c}\text { Ortopedia } \\
\text { Traumatologia }\end{array}$ & 10 & - & 2 & - & - & 3 & - & 15 \\
\hline $\begin{array}{c}\text { Cirurgia } \\
\text { Ginecológica }\end{array}$ & - & - & - & - & 16 & 2 & - & 18 \\
\hline $\begin{array}{l}\text { Otorrinolaring } \\
\text { ologista }\end{array}$ & 10 & - & - & - & - & - & - & 10 \\
\hline AIDS & 6 & - & - & - & - & - & - & 6 \\
\hline Cardiologia & 8 & - & - & - & - & - & - & 8 \\
\hline Clínica geral & 24 & 28 & 38 & 22 & 29 & 12 & 36 & 189 \\
\hline Saúde Mental & - & - & - & 8 & - & - & - & 8 \\
\hline Pneumologia & 5 & - & - & - & - & - & - & 5 \\
\hline Hospital Dia & 1 & - & - & - & - & - & - & 1 \\
\hline $\begin{array}{l}\text { Obstetrícia } \\
\text { Cirúrgica }\end{array}$ & 15 & 2 & 6 & - & - & - & 13 & 36 \\
\hline $\begin{array}{l}\text { Obstetrícia } \\
\text { Clínica }\end{array}$ & 11 & 2 & - & 1 & 20 & 2 & - & 36 \\
\hline $\begin{array}{l}\text { Pediatria } \\
\text { Clínica }\end{array}$ & 12 & 6 & 2 & 2 & 10 & 4 & 6 & 42 \\
\hline Psiquiatria & - & - & - & 10 & 1 & - & - & 11 \\
\hline $\begin{array}{l}\text { Unidade de } \\
\text { isolamento }\end{array}$ & - & - & - & - & 1 & - & - & 1 \\
\hline TOTAL & 137 & 45 & 50 & 50 & 107 & 28 & 60 & 477 \\
\hline
\end{tabular}

Fonte: Autores.

Em relação ao número de leitos por especialidade, verificou-se que $43 \%$ dos leitos são destinados à clínica médica, 19\% para ginecologia e obstetrícia, 15\% clínica cirúrgica, 9\% para pediatria, 4\% para psiquiatria e saúde mental e $10 \%$ em outras áreas. 
A importância destes achados encontra-se no fato de corresponder às áreas básicas do internato, que equivale a 70\% da carga horária obrigatória de estágio, incluindo necessariamente, aspectos essenciais das áreas de Clínica Médica, Cirurgia, Ginecologia e Obstetrícia, Pediatria, Saúde Coletiva e Saúde Mental. A carga-horária de 30\% restante pertence à MFC e urgência e emergência, conforme já discutido (Resolução nº 3, 2014).

Todavia, os resultados demonstram disparidade entre as áreas, já que algumas possuem um quantitativo pequeno de cobertura, como é o caso da pediatria e psiquiatria. Até mesmo a área de ginecologia e obstetrícia possui uma cobertura baixa na região, para atender a demanda existente.

\section{Considerações Finais}

Conclui-se que a existência de um curso de Medicina em Araranguá, sendo uma região de área rural e desassistida, é fundamental para o desenvolvimento do sistema de saúde local. Considerando as características estruturais existentes atualmente no sistema de saúde, verifica-se que a consolidação do curso de Medicina é fundamental para expandir os serviços de saúde em áreas de saúde prioritárias ao atendimento da população.

Algumas áreas que, necessariamente, precisam ser contempladas no internato médico, como a pediatria e psiquiatria, possuem quantitativo reduzido de leitos na região, necessitando expandir os serviços, como por exemplo, pela criação de programas de residência médica.

Na Atenção Básica, os resultados demonstraram que, apesar de haver um quantitativo de estabelecimentos de saúde, é necessário tomar cuidado para não haver saturação da rede de saúde. A maioria dos municípios possui UBS com uma estrutura incipiente e equipe mínima de profissionais, portanto, a contrapartida da universidade na melhoria dos serviços e do acesso é crucial.

A pesquisa revelou, ainda, que a região da Amesc oferece poucos serviços de alta complexidade até o presente momento, fato que deve, necessariamente, ser alvo de investimentos. Tanto pelo fato da entrada do curso de Medicina nos hospitais, quanto pela necessidade de oferecimento destes serviços no sentido da melhoria de acesso à população. É importante lembrar que a implantação de serviços de alta complexidade por si só não atende as demandas existentes, é também necessário investir em recursos humanos qualificados para atuar no serviço.

Destaca-se que este estudo não objetivou analisar a qualidade dos serviços prestados, tampouco o detalhamento da infraestrutura física e de recursos humanos dos estabelecimentos de saúde existentes. Em relação a demanda de serviços de saúde necessária para atender toda população da região da Amesc, não foram levantados dados suficientes para análise mais aprofundada, sugerindo que estes temas podem servir de base para pesquisas futuras.

Entende-se que, por ser uma região onde há carência de recursos de maneira geral, a entrada de estudantes de Medicina e, posteriormente, dos egressos, a médio e longo prazo, resultará no fortalecimento do sistema de saúde local. Entretanto, no momento inicial do curso, é necessário ter cuidado para não sobrecarregar os serviços de saúde com elevado número de estudantes, seja pela inserção precoce, ou pela atuação no internato médico.

Uma estratégia interessante e preconizada pelas DCN de 2014 é a implantação de programas de residência médica em MFC, que pode gerar a robustez necessária à prestação de serviços de saúde e auxiliar na supervisão dos estudantes de Medicina, além do incentivo financeiro vinculado ao programa.

Um dos desdobramentos positivos da inserção de programas de residência médica é a qualificação da preceptoria através de cursos de formação de preceptores. Entretanto, a região do extremo sul catarinense é carente de médicos com residência em MFC, sendo necessário incentivar a fixação de médicos com este perfil na região, inclusive financeiramente.

Além disso, é necessário desenvolver ações que visem contribuir para o fortalecimento da Integração Ensino-Serviço, como a pactuação através do Coapes, engajamento da universidade nas estratégias de educação permanente dos profissionais 
da rede, bem como a participação da universidade como parceira no planejamento e execução de outras políticas de saúde da região, integrando ensino, pesquisa e extensão universitárias.

\section{Referências}

Barreto, V. H. L., Monteiro, R. O. S., Magalhães, G. S. G., Almeida, R. C. C., \& Souza, L. N. (2011). Papel do preceptor da atenção primária em saúde na formação da graduação e pós-graduação da Universidade Federal de Pernambuco - um termo de referência. Revista Brasileira de Educação Médica, 35(4), 578-583. http://www.scielo.br/j/rbem/a/hyKWpJF7yhGG3PBLznnCR6g/?lang=pt\&format=pdf. 10.1590/S0100-55022011000400019

Brasil. (2004). Avaliação normativa do Programa de Saúde da Família no Brasil: monitoramento da implantação e funcionamento das equipes de saúde da família: 2001-2002. Brasília, DF: Ministério da Saúde.

Ceccim, R. B., \& Feuerwerker, L. C. M. (2004). Mudança na graduação das profissões de saúde sob o eixo da integralidade. Cad. Saúde Pública, 20(5), 14001410. Recuperado de https://www.scielosp.org/pdf/csp/v20n5/36.pdf. 10.1590/S0102-311X2004000500036

Datasus. (2020). Cadastro Nacional de Estabelecimentos de Saúde - CNES. http://cnes.datasus.gov.br/pages/estabelecimentos/consulta.jsp

Decreto $n^{\circ}$ 6.096, de 24 de abril de 2007. Institui o Programa de Apoio a Planos de Reestruturação e Expansão das Universidades Federais - REUNI. Diário Oficial da União, Brasília. http://www.planalto.gov.br/ccivil_03/decreto-lei/1965-1988/Del2423.htm

Francischetti, I., Holzhausen, Y., \& Peters H. (2020). Tempo do Brasil traduzir para a prática o Currículo Médico Baseado em Competência por meio de Atividades Profissionais Confiáveis (APCs). Interface (Botucatu), 24. https://www.scielo.br/j/icse/a/qwchcdrx4n3rxHLmFmgPPBM/?format=pdf\&lang=pt. 10.1590/Interface. 190455

Frank, J. R., Snell, L., Sherbino, J. (Eds.) (2015). Physician Competency Framework. Royal College of Physicians and Surgeons of Canada. http://canmeds.royalcollege.ca/uploads/en/framework/CanMEDS\%202015\%20Framework_EN_Reduced.pdf

IBGE. (2019). Cidades@. https://cidades.ibge.gov.br/

Lei $n^{o} 12.871$, de 22 de Outubro de 2013. Institui o Programa Mais Médicos, altera as Leis $n^{\circ} 8.745$, de 9 de dezembro de 1993 , e ${ }^{\circ}$ 6.932, de 7 de julho de 1981, e dá outras providências. http://www.planalto.gov.br/ccivil_03/_ato2011-2014/2013/lei/112871.htm

Martins, A. P. L., Negro-Dellacqua, M., Guedes, A. C. L., Souza, I.F., Biff, D., Elias, E., \& Sousa Junior, A.R. (2020). Perfil dos profissionais da Atenção Básica no Município de Araranguá/SC. Research, Society and Development, 9 (8). https://rsdjournal.org/index.php/rsd/article/view/5668. 10.33448/rsdv9i8.5668

Mendes, T. M. C., Ferreira, T. L. S., Carvalho, Y. M. C., Silva, L. G., Souza, C. M. C., \& Andrade, F.B. (2020). Contribuições e desafios da integração ensinoserviço-comunidade. Revista Texto contexto - enfermagem, 29. https://www.scielo.br/j/tce/a/KPQcPtFGXrLt4vJk76WBXrr/?format=pdf\&lang=pt. 10.1590/1980-265X-TCE-2018-0333

Ministério da Saúde, Secretaria de Gestão do Trabalho e da Educação na Saúde, Departamento de Gestão da Educação na Saúde (2018). Política Nacional de Educação Permanente em Saúde: o que se tem produzido para o seu fortalecimento? 73 p. https://bvsms.saude.gov.br/bvs/pub licacoes/politica_nacional_educacao_permanente_saude_fortalecimento.pdf

Oliveira, A.L.O., Melo, L. P., Pinto, T. R., Azevedo, G. D., \& Santos, M. (2017). Vivência integrada na comunidade: inserção longitudinal no Sistema de Saúde como estratégia de formação médica. Interface (Botucatu), 21 (Supl. 1), 1355-1365. https://www.scielo.br/j/icse/a/m3pVQcp LVpZrTCPJhXqGP6b/?format=pdf\&lang=pt. 10.1590/1807-57622016.0533

Pedrosa, J. I. S. (2019). Implantação e desenvolvimento do curso de Medicina em Parnaíba (PI), Brasil, a partir do Programa Mais Médicos para o Brasil. Interface (Botucatu), 23 (Supl. 1), 1-15. https://www.scielo.br/j/icse/a/njfkR9CT7mWt3QDZBpkTbcC/?lang=pt\&format=pdf. 10.1590/Interface.180012

Portaria Interministerial $n^{\circ}$ 1.127, de 04 de agosto de 2015. Institui as diretrizes para a celebração dos Contratos Organizativos de Ação Pública Ensino-Saúde (COAPES), para o fortalecimento da integração entre ensino, serviços e comunidade no âmbito do Sistema Único de Saúde (SUS). http://portalarquivos.saude.gov.br/images/pdf/2015/outubro/23/COAPES-PORTARIA-INTERMINISTERIAL-N1.127\%20-DE-04\%20DE-AGOSTO-DE2015.pdf

Resolução $n^{o}$ 3, de 20 de junho de 2014. Institui diretrizes curriculares nacionais do curso de graduação em medicina e dá outras providências. http://portal.mec.gov.br/index.php?option=com_docman\&view=download\&alias=15874-rces003 14\&category_slug=junho-2014-pdf\&Itemid=30192

Resolução $n^{o} 466$, de 12 de dezembro de 2012. Dispõe sobre aprovação de diretrizes e normas regulamentadoras de pesquisas envolvendo seres humanos. http://conselho.saude.gov.br/resolucoes/2012/Reso466.pdf

Rocha, E. M. S., Boiteux, P. A., Azevedo, G. D., Siqueira, C. E. G., \& Andrade, M. A. C. (2020). Preditores Educacionais para Fixação de Médicos em Áreas Remotas e Desassistidas: uma Revisão Narrativa. Revista Brasileira de Educação Médica, 44(1). https://www.scielo.br/j/rbem/a/Z7J6wW 9W78hHkdTZkbQ7Bkk/?format=pdf\&lang=pt. 10.1590/1981-5271v44.1-20190281

Rouquayrol, Z., Gurgel, M. Epidemiologia \& Saúde. (7a ed.), Editora Medbook, 2013.

Secretaria de Estado da Saúde (2020). Hospital Regional de Araranguá e Policlínica de Araranguá - Organização Social IMAS. https://www.saude.sc.gov.br/index.php/documentos/informacoes-gerais/organizacoes-sociais/contratos-de-gestao/contratos-de-gestao-vigentes/c-g-004-2018hospital-regional-de-ararangua-e-policlinica-de-ararangua-organizacao-social-imas

Shipley, T. F., \& Cunningham, D. W. (2001). Perception of occluding and occluded objects over time: Spatiotemporal segmentation and unit formation. Adv Psychol. 130(C), 57-85. https://psycnet.apa.org/record/2002-00597-018 
Research, Society and Development, v. 10, n. 10, e139101018767, 2021

(CC BY 4.0) | ISSN 2525-3409 | DOI: http://dx.doi.org/10.33448/rsd-v10i10.18767

SIM. (2012). Sistema de Informação sobre Mortalidade. Recuperado de https://ces.ibge.gov.br/base-de-dados/metadados/ministerio-da-saude/sistema-deinformacoes-de-mortalidade-sim.html

Sociedade Brasileira de Medicina de Família e Comunidade. (2019). Nota sobre a nova política de financiamento da atenção primária à saúde - Programa Previne Brasil. https://www.sbmfc.org.br/noticias/nota-sobre-nova-politica-de-financiamento/

Word Federation for Medical Education (2003) (Rev. Ed.). Global Standards for Quality Improvement. https://www.um.es/documents/1935 287/1936044/Revision_of_Standards_for_Basic_Medical_Education_FINAL_260912.pdf/5866f7af-f7fc-4f9a-a7e6-eb054b7795c3 